\title{
ANAESTHETIC MANAGEMENT OF A PATIENT WITH COMPLICATED MALIGNANT OTITIS EXTERNA, VOCAL CORD PALSY AND MULTIPLE LEVEL INTERVERTEBRAL DISC PROLAPSE
}

\author{
Leena Harshad Parate ${ }^{1}$, Shivakumar Shivanna², Manjunath A. C³, Vinayak S Pujari4, Renu Joy5.
}

\footnotetext{
1. Assistant Professor, Department Of Anaesthesia, M S Ramaiah Medical College And Hospitals

2. Professor, Department Of Anaesthesia, M S Ramaiah Medical College And Hospitals

3. Associate Professor, Department Of Anaesthesia, M S Ramaiah Medical College And Hospitals

4. Associate Professor, Department Of Anaesthesia, M S Ramaiah Medical College And Hospitals

5. Junior Resident, Department Of Anaesthesia, M S Ramaiah Medical College And Hospitals
}

\section{CORRESPONDING AUTHOR:}

Dr. Shivakumar Shivanna, M S Ramaiah Medical College and Hospitals.

Bangalore560043.

E-mail: shivanna03@gmail.com

\section{HOW TO CITE THIS ARTICLE:}

Leena Harshad Parate, Shivakumar Shivanna, Manjunath A.C, Vinayak S Pujari, Renu Joy. "Anaesthetic Management of a Patient with complicated Malignant Otitis Externa, Vocal Cord Palsy and Multiple Level Intervertebral Disc Prolapse". Journal of Evolution of Medical and Dental Sciences 2013; Vol2, Issue 26, July 1; Page: $4747-4751$.

ABSTRACT: An elderly male patient with Malignant Otitis Externa (MOE) and Polyneuritis Cranialis Multiplex (PCM) presented with chronic osteomyelitis of left foot for Ilizarov application. This case represents a classic case of MOE with significant complications. Intra cranially VI, VII, VIII, IX and X cranial nerves were involved and the patient had intervertebral disc prolapse in the cervical and lumbar region with right vocal cord palsy. Considering multiple cranial nerve palsies and multiple lumbar intervertebral disc prolapses we preferred peripheral nerve block for the surgery. This case was successfully managed with ultrasound guided (USG) popliteal nerve and femoral nerve block.

KEY-WORDS: Malignant Otitis Externa, Polyneuritis Cranialis Multiplex, ultrasound guided nerve block

INTRODUCTION: MOE is a rare, life threatening complication commonly seen in long standing diabetics. It results from spread of infection from external auditory canal to the skull base. In the advanced stage it affects multiple cranial nerves, temporomandibular joint, parapharyngeal space and meninges. Here we report a case of MOE with PCM who underwent lower extremity Ilizarov application under USG guided popliteal and femoral nerve block.

CASE REPORT: A $64 \mathrm{yr}$ old male patient was admitted with history of dysphagia, diplopia, hoarseness of voice and non healing ulcer over the left foot. Previously he underwent right mastoidectomy and debridement of skull base granulation tissue. He was a known diabetic and hypertensive for the last 20yrs for which he was on antihypertensives and oral hypoglycemic agents. On examination, the patient was afebrile, conscious, oriented and haemodynamically stable. 
Neurological examination revealed right eye diplopia, right upper motor neuron(UMN) facial palsy, right conductive deafness with absent gag reflex corresponding to VI,VII, VIII, IX and X nerve palsy. The muscle power was 4/5 in both upper and lower limbs with intact deep tendon reflexes. Sensory system was apparently normal and no cerebellar signs. Airway examination revealed bilateral subluxation of temporomandibular joint (TMJ) with mild restriction of neck extension.

Indirect laryngoscopy revealed right vocal cord palsy and edema of vocal cords involving arytenoid cartilages. Computerized tomography (CT) brain with contrast showed communication between right middle ear cavity and middle cranial fossa with skull base destruction involving anterior arch of C1 vertebrae.MRI spine showed diffuse disc bulge at C5-C6, L3-L4, L4-L5 and L5-S1 level with posterior annular tear indenting thecal sac anteriorly at L4-L5 level. Open mouth cervical spine $\mathrm{X}$ ray showed cervical spondylosis with C5-C6 disc disease.

Nerve conduction study showed prolonged latency, reduced amplitude and conduction velocity of left common peroneal nerve and bilateral tibial nerve. On sensory conduction study, there was absent sensory nerve action potential (SNAP) seen in both sural nerves.CSF examination showed raised glucose and protein levels and culture sensitivity showed no growth. Wound culture showed growth of S. aureus for which IV ceftriaxone was started. Electrocardiography, echocardiography and haematological and biochemical investigations were normal. Biochemical investigations revealed creatinine of $1.4 \mathrm{mg} \%$, blood urea $12 \mathrm{mg} \%$ and potassium of $4.5 \mathrm{meq} / \mathrm{L}$

He was diagnosed to have PCM secondary to MOE with chronic osteomyelitis of left foot. He was scheduled to undergo Ray's amputation of $4^{\text {th }}$ and $5^{\text {th }}$ digit with Ilizarov application to left lower limb. Preoperatively, the patient was commenced on maintenance intravenous dextrose normal saline with insulin and a tight glycemic control was established. In view of PCM, right vocal cord palsy and intervertebral disc prolapse of multiple lumbar segments anaesthetic plan was USG guided popliteal and femoral nerve block. Consent was also obtained for caudal epidural anaesthesia as the second plan in case the USG guided popliteal and femoral nerve block failed. The patient was explained about his diabetic neuropathy status and written anaesthetic consent obtained. The patient was kept nil per orally for 6 hours and premedicated with Ondansetron $4 \mathrm{mg}$ and Pantoprazole $40 \mathrm{mg} 60$ minutes prior to the surgery. Before the procedure, the patient received IV midazolam $1 \mathrm{mg}$ and fentanyl $25 \mu \mathrm{g}$ and supplemental oxygen administered via face mask. In the theatre patient was positioned in the right lateral position. After local anaesthetic infiltration, the stimuplex needle tip was correctly placed near popliteal nerve under USG guidance and 20cc of $0.375 \%$ ropivacaine with 90 ug clonidine was injected (Fig 1) around the nerve. The femoral nerve was identified in supine position with USG guidance $15 \mathrm{cc}$ of $0.375 \%$ ropivacaine was administered. The surgery commenced after loss of pin prick and cold sensation, and lasted for $1 \mathrm{hr}$. Intraoperative blood sugars was controlled with intravenous insulin as per sliding scale. Postoperatively the patient received IV paracetamol for analgesia.

DISCUSSION: MOE is a term first coined by JR Chandler in 1968 to describe the severity of the infection of external auditory canal ${ }^{1}$. It is also known as necrotizing otitis externa or skull base osteomyelitits.PCM is a rare and serious complication of malignant otitis externa (MOE) ${ }^{2}$. This is commonly seen in diabetic and elderly patients. In non diabetic patients it usually affects immunocompromised patients secondary to malignancy, HIV, malnutrition and chemotherapy. Pseudomonas aeruginosa are most common cause of MOE followed by S. Aureus and fungal 


\section{CASE REPORT}

infection ${ }^{2}$ Such patients needing various surgeries may require special consideration while planning anaesthesia.

MOE results from spread of infection from external auditory canal to skull base. Skull base osteomyelitis with intracranial spread has a significant morbidity and mortality rate. Intracranial complications consist of multiple cranial neuropathy, cerebral abscess, dural sinus thrombosis and meningitis ${ }^{2}$ 3.Neurotoxin released by microorganisms and subsequent skull base inflammation isproposed mechanism behind cranial neuropathy ${ }^{4}$.Incidence of PCM has been shown to be 43.5\% in the patients with MOE ${ }^{5}$.The most commonest nerve involved is VII nerve at the stylomastoid foramen. As the infection spreads to the apex of petrous bone V and VI nerves are affected while IX, $\mathrm{X}, \mathrm{X}$ nerve are affected via the jugular foramen. Except for $1^{\text {st }}$ cranial nerve all nerves can be affected 3.In advanced stage even contralateral nerves can get affected ${ }^{6}$. Saha et al reported a case of MOE with PCM who required thyroplasty for vocal cord to control chronic aspiration and to improve quality of voice. Septic thrombosis of internal jugular vein and carotid artery can lead to meningitis and cerebral infarction respectively ${ }^{7}$.

Extracranial complications are seen due to the spread of infection to the cervical spine, nasopharynx, carotid canal and TMJ. The incidence of TMJ involvement has been shown to be $14 \%$ and represents resistant disease process ${ }^{8,9}$. The TMJ osteomyelitis is a very rare complication of MOE which lead to abscess formation, subluxation ${ }^{10}$ and in extreme case destruction of mandibular condyle7,8. It may require surgical debridement of TMJ ${ }^{11}$ and correction with prosthesis. Dobbyn et al reported a case where MOE had invaded right TMJ which required replacement of glenoid fossa with a Silastic prosthesis ${ }^{12}$. CT scan remains the initial investigation of choice to define anatomical extension and soft tissue involvement of the disease. MRI, Technetium 99, Gallium 67 scintigraphy can give additional information about disease progress ${ }^{2}$.

Our case represents a classic case of MOE with intracranial and extracranial complications. Such patients are potential challenges for anesthesiologists. To our knowledge there is no literature providing information about anaesthetic considerations in such set of patients. In our patient we preferred peripheral regional block since it was a lower limb surgery. We avoided general anaesthesia as well as central neuraxial block since patient had various problems related to MOE, in addition to multiple level intervertebral disc disease. In the patients requiring general anaesthesia special consideration should be given to preoperative evaluation of airway. TMJ and cervical spine involvement should be ruled out with necessary radiological evaluation. Pharyngeal cellulitis, edematous airway, vocal cord palsy, loss of gag reflex is areas of concern while securing the airway.

Our patient had VI, VII, VIII, IX and X cranial nerve palsies with no signs of active intracranial infection. Although CT scan revealed erosion of the mandibular condyle, symptoms of TM joint osteomyelitis such as tenderness, masseter spasm were absent. He had right recurrent laryngeal nerve palsy, loss of gag reflex which are potential challenges for securing the airway under general anaesthesia. Skull base osteomyelitis had involved C1 vertebrae in addition to C5-C6 disc disease which will need special attention during airway manipulation. Joseph A. first reported a case of MOE causing destruction of upper cervical vertebrae. Cervical spine involvement may occur through surrounding skull base osteomyelitis or via anterior longitudinal ligaments of vertebrae ${ }^{13}$.Yaeeun Suh reported a case where MOE resulted in odontoid destruction and spondylo discitis and fusion of C5-6 vertebrae resulting from haematogenous spread of infection ${ }^{14}$.USG guided popliteal nerve block seems to be the best choice for providing anaesthesia for these cases. 
Peripheral nerve block causes least systemic haemodynamic changes and provides good postoperative pain relief. USG improves efficacy of the block by direct visualization of nerve, spread of local anaesthetic simultaneously reducing dose of drug ${ }^{15}$.

CONCLUSION: In the present case, the patient had MOE with PCM involving cervical spine and intervertebral disc prolapse of multiple segments. We managed this case successfully with USG guided popliteal nerve and femoral nerve block. In such patients when general anaesthesia or central neuraxial block is necessary thorough workup with multidisciplinary approach is mandatory.

\section{REFERENCES:}

1. Chandler JR. Malignant external otitis. Laryngoscope, 1968, 78:1257-94.

2. Carfrae MJ, Kesser BW. Malignant otitis externa. Otolaryngol clin N Am. 2008; 41:537-49.

3. Singh A, Al Khabori M, Hyder MJ. Skull base osteomyelitis. Diagnostic and therapeutic challenges in atypical presentation. Otolaryngol Head Neck Surg. 2005; 133(1):121-5.

4. Illing E, Olaleye O. Malignant Otitis Externa: A Review of Aetiology, Presentation, Investigations and Current Management Strategies. Webmed Central Otorhinolaryngology. 2011; 2(3):WMC001725.

5. Mani N, Sudhoff H, Rajagopal S, Moffat D, Axon PR. Cranial nerve involvement in malignant external otitis: Implications for clinical outcome. Laryngoscope 2007; 117:907-10.

6. Saha S, Chowdhury K, Pal S, Saha VP. Malignant otitis externa with bilateral cranial nerve involvement: Report of a unique case. Indian J Otol. 2013;19:33-5

7. Po-yu liv, Zhi-yuan shi, Wyane Huey-Herng Sheu. Malignant otitis externa in patients with Diabetes Mellitus. Formos. J. Endocrin Metab. 2012; 3 (1): 7-13.

8. Mardinger O, Rosen D, Minkow B, Tulzinsky Z, Ophir D, Hirshberg A. Temporomandibular joint involvement in malignant external otitis. Oral Surg Oral Med Oral Pathol Oral Radiol Endod. 2003; 96(4):398-03.

9. Midwinter KI, Gill KS, Spencer JA, Fraser DI. Osteomyelitis of the temporomandibular joint in patients with malignant otitis externa. The Journal of Laryngology \& Otology, 1999; 113(05): 451-53.

10. Duvvi SK, Lo S, Kumar R, Blanshard J. Malignant External Otitis with Multiple Cranial Nerve Palsies: The Use Of Hyperbaric Oxygen. The Internet Journal of Otorhinolaryngology. 2005 ; 4 :Number 1. DOI: $10.5580 / 22 a 7$.

11. Ebenezer R, Khan F, Nair S, Sajilal M. Malignant otitis externa. An unusual presentation. Indian J Otol, 2011; 3: 127-29.

12. Dobbyn L, O. Shea C, Mcloughlin P. Malignant otitis externa involving the temporomandibular joint. Journal of laryngology and otology, 2005;119:61-63

13. Giordano J, Phillips J. Malignant External Otitis, Now a Medical Problem. Diabetes care,1980;3:611-14

14. Suh Y, Ilyas S, Bejjanki NK, Al-Nammari S. Otitis Externa - what a pain in neck ; Scott Med J 2007; 52:453. 


\section{CASE REPORT}

15. Van Geffen GJ, Van den Broek E, Braak GJ, Giele JL, Gielen MJ, Scheffer GJ. A prospective randomised controlled trial of ultrasound guided versus nerve stimulation guided distal sciatic nerve block at the popliteal fossa. Anaesth Intensive Care. 2009; 37(1):32-7.

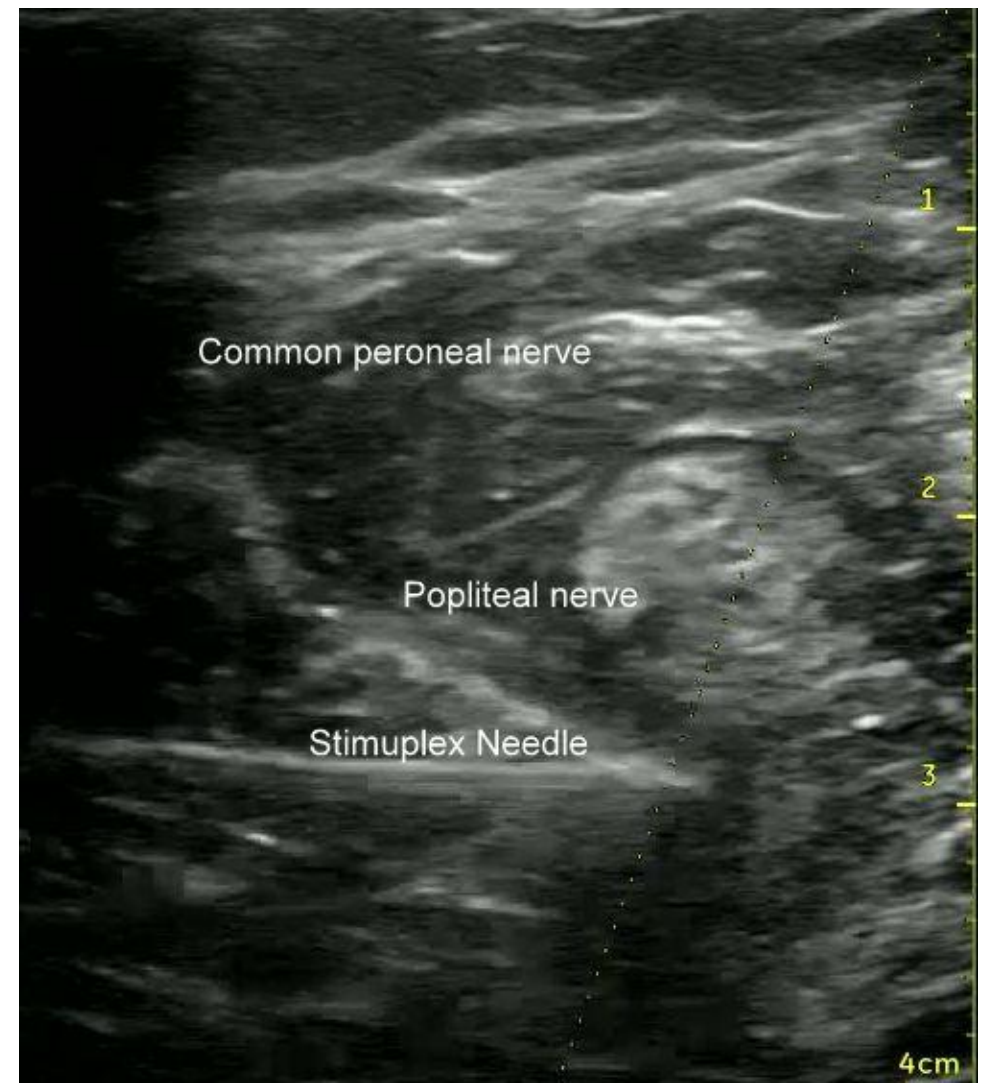

Fig-1 Popliteal Nerve block being performed under Ultrasound Guidance 